\title{
Large Anomalous Hall Effect in Topological Insulators with Proximitized Ferromagnetic Insulators
}

\author{
Masataka Mogi, ${ }^{1, *}$ Taro Nakajima, ${ }^{2}$ Victor Ukleev, ${ }^{2,3}$ Atsushi Tsukazaki, ${ }^{4}$ Ryutaro Yoshimi, ${ }^{2}$ \\ Minoru Kawamura, ${ }^{2}$ Kei S. Takahashi, ${ }^{2,5}$ Takayasu Hanashima, ${ }^{6}$ Kazuhisa Kakurai, ${ }^{2,6}$ Taka-hisa Arima, ${ }^{2,7}$ \\ Masashi Kawasaki, ${ }^{1,2}$ and Yoshinori Tokura ${ }^{1,2,8, \dagger}$ \\ ${ }^{1}$ Department of Applied Physics and Quantum Phase Electronics Center (QPEC), University of Tokyo, \\ Bunkyo-ku, Tokyo 113-8656, Japan \\ ${ }^{2}$ RIKEN Center for Emergent Matter Science (CEMS), Wako, Saitama 351-0198, Japan \\ ${ }^{3}$ Laboratory for Neutron Scattering and Imaging (LNS), Paul Scherrer Institute (PSI), CH-5232, Villigen, Switzerland \\ ${ }^{4}$ Institute for Materials Research, Tohoku University, Sendai, Miyagi 980-8577, Japan \\ ${ }^{5}$ PRESTO, Japan Science and Technology Agency (JST), Chiyoda-ku, Tokyo 102-0075, Japan \\ ${ }^{6}$ Comprehensive Research Organization for Science and Society (CROSS), Tokai, Ibaraki 319-1106, Japan \\ ${ }^{7}$ Department of Advanced Materials Science, University of Tokyo, Kashiwa, Chiba 277-8561, Japan \\ ${ }^{8}$ Tokyo College, University of Tokyo, Bunkyo-ku, Tokyo 113-8656, Japan
}

(Received 30 November 2018; revised manuscript received 24 March 2019; published 2 July 2019)

\begin{abstract}
We report a proximity-driven large anomalous Hall effect in all-telluride heterostructures consisting of the ferromagnetic insulator $\mathrm{Cr}_{2} \mathrm{Ge}_{2} \mathrm{Te}_{6}$ and topological insulator $(\mathrm{Bi}, \mathrm{Sb})_{2} \mathrm{Te}_{3}$. Despite small magnetization in the $(\mathrm{Bi}, \mathrm{Sb})_{2} \mathrm{Te}_{3}$ layer, the anomalous Hall conductivity reaches a large value of $0.2 e^{2} / h$ in accord with a ferromagnetic response of the $\mathrm{Cr}_{2} \mathrm{Ge}_{2} \mathrm{Te}_{6}$. The results show that the exchange coupling between the surface state of the topological insulator and the proximitized $\mathrm{Cr}_{2} \mathrm{Ge}_{2} \mathrm{Te}_{6}$ layer is effective and strong enough to open the sizable exchange gap in the surface state.
\end{abstract}

DOI: 10.1103/PhysRevLett.123.016804

In magnetically doped three-dimensional (3D) topological insulator (TI) films, exotic magnetic quantum phases such as a quantum anomalous Hall (QAH) insulator and an axion insulator have been achieved [1-11]. The formation of an exchange gap at the Dirac surface states of TI films and the Fermi-level tuning into the gap are two requisites for the emergence of the topological phenomena, e.g., the chiral edge conduction in the QAH state. In the magnetically doped TI, the size of the energy gap ( $\sim 50 \mathrm{meV}[12])$ is produced by the interaction between magnetic impurities and the surface states, whereas it suffers from disorders due to spatial inhomogeneity of magnetic dopants $[12,13]$ and electronic potentials [14]. In fact, the observable temperature of the QAH effect reported so far is lower than about $100 \mathrm{mK}$ in the uniformly $\mathrm{Cr}$ - or V-doped $(\mathrm{Bi}, \mathrm{Sb})_{2} \mathrm{Te}_{3}$ films $[5,6]$. The modulation doping or codoping technique of the magnetic ions has been developed to reduce the disorder, yet the observable QAH temperature still remains at most around $2 \mathrm{~K}[7,8]$. The ferromagnetic proximity effect is anticipated to be an alternative ideal approach to introduce the uniform magnetic interaction to the surface states [1-3]. The choice of materials for ferromagnetic insulators (FMIs) is a key issue to induce the effective coupling with less disorder; candidates for the FMIs facing the TI are of a great variety. Indeed, several FMI-TI heterostructures have been proposed theoretically and synthesized to date [15-29]. Although these studies have demonstrated several potential magnetoelectronic responses, such as magnetoresistance, the anomalous Hall effect [17-24], and unconventional surface magnetization, [25,26] even at room temperature, the magnitude of the response or the coupling strength to the surface state of the TI remains far smaller than expected.

In this Letter, we report a large anomalous Hall effect, being reminiscent of an incipient QAH state, in a FMI-TI heterostructure consisting of $\mathrm{Cr}_{2} \mathrm{Ge}_{2} \mathrm{Te}_{6}$ (CGT) [21,30-33] and $(\mathrm{Bi}, \mathrm{Sb})_{2} \mathrm{Te}_{3}$ (BST) [5-13,22,23,26]. The observation indicates the formation of a sizable exchange gap in the surface state of the TI. Through combined characterizations of the interfacial magnetic property by spin-polarized neutron reflectometry and by magnetotransport measurements, we demonstrate that the exchange coupling is induced by the magnetic proximity effect.

We fabricated the CGT/BST heterostructures on InP(111) substrates by molecular-beam epitaxy (MBE) (see the Supplemental Material [34] for detailed methods). Ferromagnetic CGT thin layers have recently been achieved, not only by mechanical exfoliation of bulk crystals [32], but also by thin film growth with MBE [33]. CGT has a rhombohedral crystal structure of a van der Waals ( $\mathrm{vdW}$ ) type [30], which is presumably matched to the interface formation with a similar triangular lattice constant of BST [Fig. 1(b)] [21,31]. Furthermore, it has been reported that the MBE-grown CGT films possess a perpendicular anisotropic 
(a)

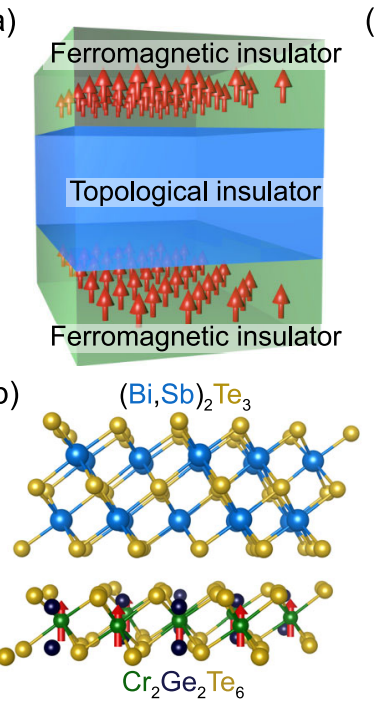

(c)

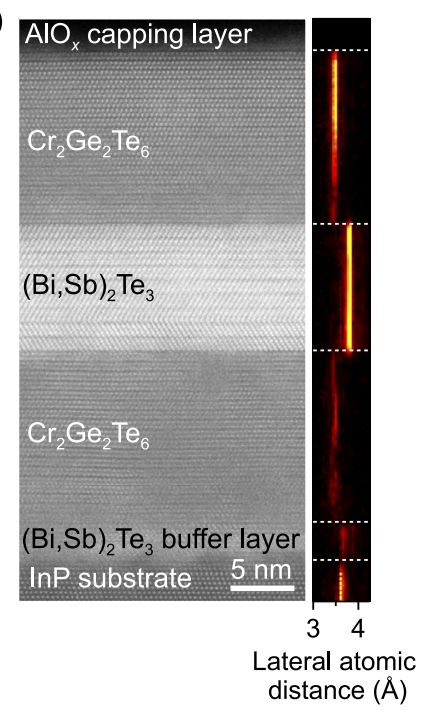

(a)

(c)
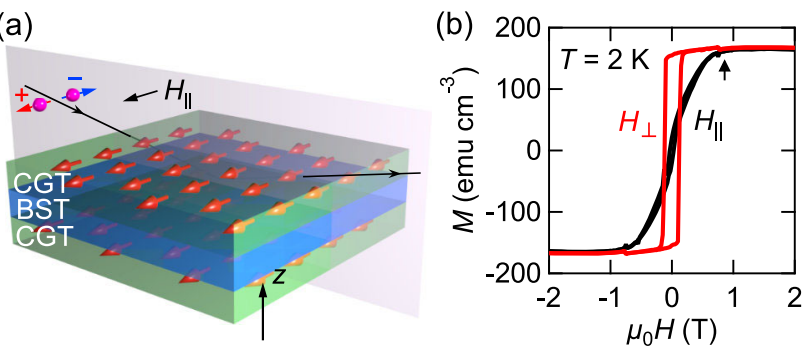

FIG. 1. (a) Schematic drawing of the interfacial exchange coupling in a 3D TI sandwiched by FMIs. (b) Schematic of crystal structures of $\mathrm{TI}(\mathrm{Bi}, \mathrm{Sb})_{2} \mathrm{Te}_{3}$ and $\mathrm{FMI} \mathrm{Cr}_{2} \mathrm{Ge}_{2} \mathrm{Te}_{6}$ with the relationship of stacking orientation expected from their $\mathrm{Te}$ arrangements in the respective layer planes. (c) Cross-sectional high-angle annular dark-field STEM image of the CGT(12 nm)/ $\operatorname{BST}(9 \mathrm{~nm}) / \mathrm{CGT}(12 \mathrm{~nm})$ heterostructure grown on an InP substrate with a BST buffer layer (left). The lateral atomic distance of each layer obtained by the Fourier transformation of the left image plotted along the growth direction (right).

remanent magnetization [33,34], which is favorable to produce the exchange gap in the surface state of the TI. Experimentally, the structural characterization of the interface was carried out by a cross-sectional scanning transmission electron microscopy (STEM). Figure 1(c) displays the STEM image of a MBE-grown CGT(12 nm)/ $\operatorname{BST}(9 \mathrm{~nm}) / \operatorname{CGT}(12 \mathrm{~nm})$ heterostructure, exhibiting abrupt interfaces with the ordered stacking orientation in favor of the hexagonal Te arrangements of CGT and BST. By performing Fourier transformation in the lateral direction of the image, the lattice distance of each layer is achieved as depicted in the right panel of Fig. 1(c). Sharp changes of the lateral lattice distance at the interfaces reflect weak epitaxial strain at the interfaces, which are a notable feature of the $\mathrm{vdW}$ heterointerface. Furthermore, energy-dispersive x-ray spectroscopy ensures almost no interdiffusion of atoms [34].

On the basis of the MBE-grown clean heterostructures, we examine the interfacial magnetism of the CGT/BST/ CGT sandwiched heterostructure by depth-sensitive polarized neutron reflectometry (PNR) [34]. The PNR measurements, being quantitatively responsive to in-plane magnetization, were conducted at $3 \mathrm{~K}$ with an in-plane magnetic field $\mu_{0} H_{\|}=1 \mathrm{~T}$ [Fig. 2(a)], which is strong enough to fully align the magnetic moments to the field direction as confirmed by the magnetization hysteresis loops measured at $2 \mathrm{~K}$ [Fig. 2(b)]. Figure 2(c) shows the x-ray and nonspin-flip specular PNR reflectivity curves $R^{+}$
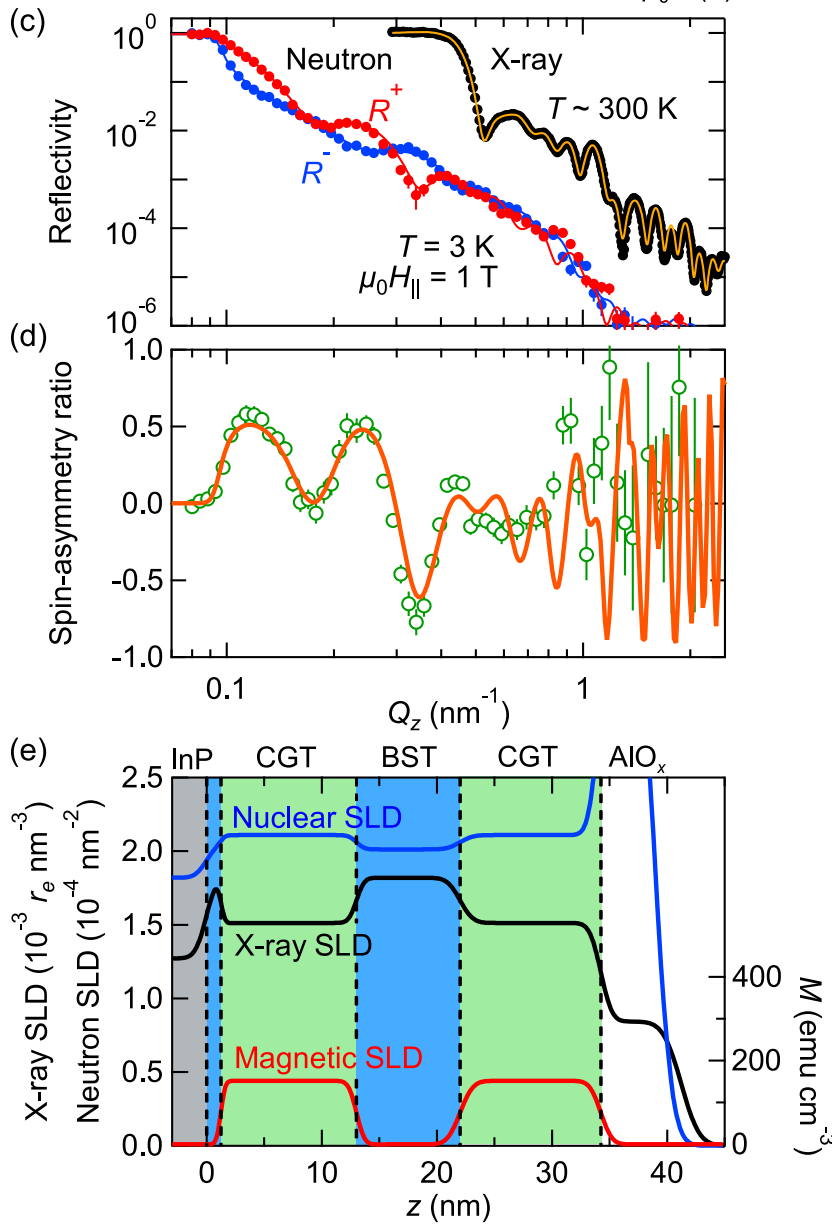

FIG. 2. (a) Schematic of the PNR experimental setup for the CGT/BST/CGT structure. (b) Magnetization hysteresis loops under out-of-plane $\left(H_{\perp}\right)$ and in-plane $\left(H_{\|}\right)$magnetic fields for the identical CGT(12 nm)/BST(9 nm)/CGT(12 nm) sample used in the PNR experiments. The black arrow represents the saturation field for the in-plane direction. (c) Measured (dots) and fitted (solid lines) reflectivity curves for the x-ray (black) and neutron of spin-up $\left(R^{+}\right)$(red) and spin-down $\left(R^{-}\right)$(blue) as a function of momentum transfer $\left(Q_{z}\right)$ on a logarithmic scale. The error bars represent one standard deviation. (d) PNR spinasymmetry ratio $\left(R^{+}-R^{-}\right) /\left(R^{+}+R^{-}\right)$obtained from experimental and fitted reflectivity curves in (c). The error bars represent one standard deviation. (e) X-ray SLD (black) and neutron SLD divided into the nuclear (blue) and the magnetic (red) SLDs as a function of the distance from the InP substrate surface $(z)$. The $r_{e}$ in the unit of the x-ray SLD denotes the classical electron radius of $2.8179 \ldots \times 10^{-15} \mathrm{~m}$. For the magnetic SLD, the value of $M$ corresponding to the neutron SLD is shown in the right ordinate. 
and $R^{-}$, where $+(-)$denotes the incident neutron spins parallel (antiparallel) to the direction of $H_{\|}$as a function of the momentum transfer vector $Q_{z}$. The in-plane saturated magnetization of the sample is directly reflected in the spin-asymmetry ratio defined as $\left(R^{+}-R^{-}\right) /\left(R^{+}+R^{-}\right)$ [Fig. 2(d)]. In addition, we combined an x-ray reflectivity (XRR) measurement at room temperature [Fig. 2(c)] to conduct a model analysis for the structural parameters, including thickness and roughness of each layer. The depth profile of the x-ray scattering length density (SLD) shown in Fig. 2(e), corresponding to the electron density distribution in the heterostructure, reflects the structural interface roughness. Notably, the root-mean-square roughness of all interfaces in the SLD profiles is less than $1 \mathrm{~nm}$, which is consistent with the STEM image shown in Fig. 1(c). The structural parameters derived from the XRR fitted model were used to refine the PNR curves. Figure 2(e) displays the magnetic SLD depth profiles based on the fitting results on $R^{+}, R^{-}$[Fig. 2(c)], and spin-asymmetry ratio [Fig. 2(d)], taking into account the structural depth profile obtained from the XRR data. The fitting analysis yields magnetizations of $152 \pm 8$ and $0 \pm 20 \mathrm{emu} / \mathrm{cm}^{3}$ for the CGT and BST layers [34], respectively. Although it is difficult to precisely determine the induced magnetization in the BST layer due to spatial broadening, it will be reasonable to conclude from the present fitting analysis that the induced magnetization in the BST layer is far smaller than the magnetization of the CGT layer.

The ferromagnetic proximity effect on the surface states of the TI can be assessed by magnetotransport measurements. The measurements were conducted with the sandwiched CGT/BST/CGT trilayers and the CGT/BST bilayers. Because of the high electric resistance of the CGT layer [33], its contribution to electrical transport is negligibly small [34]. For the TI layer, instead of simple single-layered $\left(\mathrm{Bi}_{1-x} \mathrm{Sb}_{x}\right)_{2} \mathrm{Te}_{3}$, we engineered a multilayer structure of $\left(\mathrm{Bi}_{1-x} \mathrm{Sb}_{x}\right)_{2} \mathrm{Te}_{3}(2 \mathrm{~nm}) / \mathrm{Bi}_{2} \mathrm{Te}_{3}(2 \mathrm{~nm}) /\left(\mathrm{Bi}_{1-x} \mathrm{Sb}_{x}\right)_{2} \mathrm{Te}_{3}(2 \mathrm{~nm})$ [Fig. 3(a)], which works as a conduction channel. The reason for adopting the multilayer structure is as follows. In the CGT/BST/CGT heterostructures, the charge neutrality point takes place at a relatively small value of $x(0.3<$ $x<0.4$ ) due to possible hole transfer from CGT to BST [34]. According to an angle-resolved photoemission spectroscopy study on BST [41], small $x$ causes the Dirac point to submerge below the bulk valence band. To approach the Dirac point with the charge neutrality condition, we need to dope electrons while keeping $x>0.5$. To fulfill this requirement, we inserted the electron-rich $\mathrm{Bi}_{2} \mathrm{Te}_{3}$ layer between the BST layers to assist electron doping. The value of $x$ in the BST layer is kept larger than 0.5, assuming that the surface band structure is mainly affected by the environment near the interface [36]. Consequently, we could prepare the samples with low carrier density at reasonably large $\mathrm{Sb}$ compositions, $x=0.6$ and 0.64 , which show semiconducting temperature $(T)$ dependence of the longitudinal sheet resistivity $\left(\rho_{x x}\right)$ as depicted in Fig. 3(b).

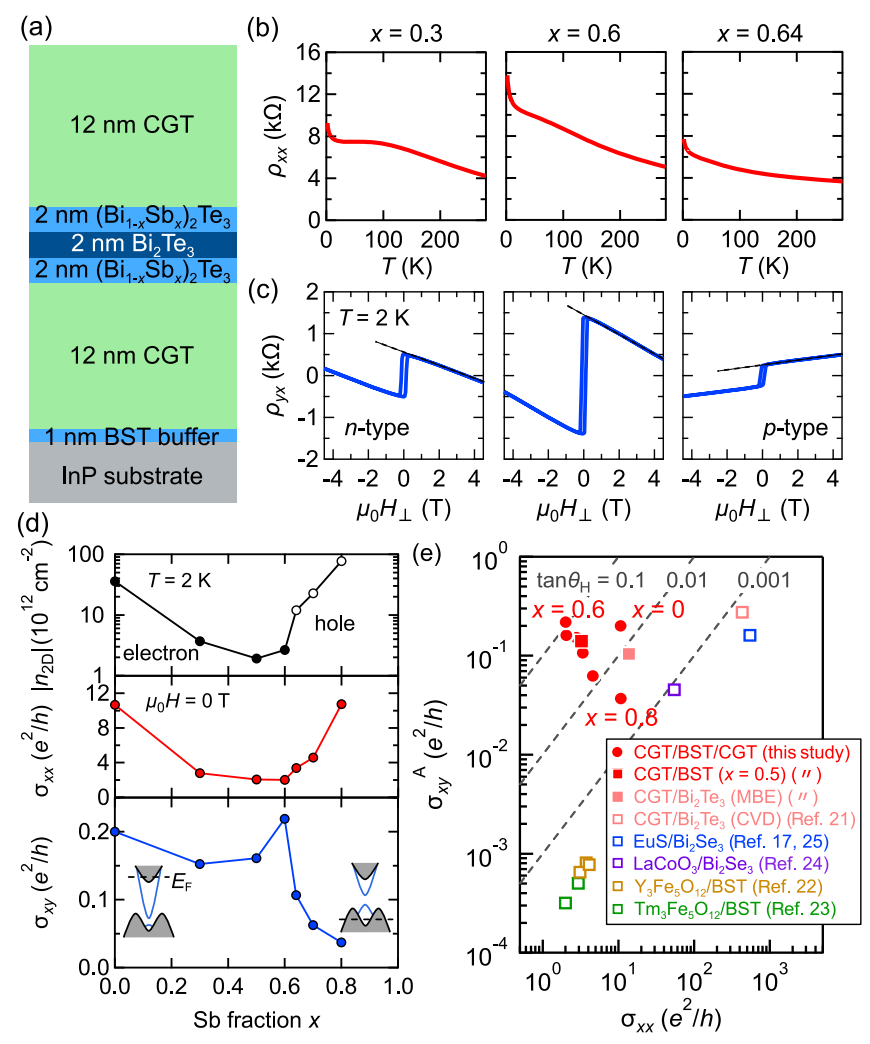

FIG. 3. (a) Schematic layout of $\mathrm{CGT}(12 \mathrm{~nm}) /\left(\mathrm{Bi}_{1-x} \mathrm{Sb}_{x}\right)_{2}$ $\mathrm{Te}_{3}(2 \mathrm{~nm}) / \mathrm{Bi}_{2} \mathrm{Te}_{3}(2 \mathrm{~nm}) /\left(\mathrm{Bi}_{1-x} \mathrm{Sb}_{x}\right)_{2} \mathrm{Te}_{3}(2 \mathrm{~nm}) / \mathrm{CGT}(12 \mathrm{~nm})$ heterostructure. (b), (c), Temperature $(T)$ [out-of-plane magnetic field $\left(\mu_{0} H_{\perp}\right)$ ] dependence of the longitudinal sheet resistivity $\left(\rho_{x x}\right)$ in zero-field (b) [the Hall resistivity $\left(\rho_{y x}\right)$ at $2 \mathrm{~K}(\mathrm{c})$ ] of $\mathrm{CGT} /\left(\mathrm{Bi}_{1-x} \mathrm{Sb}_{x}\right)_{2} \mathrm{Te}_{3} / \mathrm{Bi}_{2} \mathrm{Te}_{3} /\left(\mathrm{Bi}_{1-x} \mathrm{Sb}_{x}\right)_{2} \mathrm{Te}_{3} / \mathrm{CGT} \quad(x=0.3$, $0.6,0.64)$ heterostructures. (d) Sb fraction $(x)$ dependence of the sheet carrier density $\left(\left|n_{2 D}\right|\right)$ (top), the longitudinal sheet conductivity $\left(\sigma_{x x}\right)$ (middle), and the Hall conductivity $\left(\sigma_{x y}\right)$ (bottom) at $2 \mathrm{~K}$. (Insets) Simplified schematics of band structures representing the different Fermi energies; the blue lines represent the dispersion of the surface state. (e) The anomalous Hall conductivity $\sigma_{x y}{ }^{A}$ is plotted against the $\sigma_{x x}$ plot, with the use of the data for the present heterostructures shown in (d) in comparison with other various FMI-TI heterostructures [17,21-25]. The values of $\sigma_{x y}{ }^{A}$ and $\sigma_{x x}$ are taken from the data obtained at the lowest temperature in the measurements $(2-5 \mathrm{~K})$ for the magnetized state where the out-of-plane magnetization saturates.

In these samples, large anomalous Hall resistance $(>1 \mathrm{k} \Omega$ ) appears with perpendicular anisotropic hysteresis loops as shown in Fig. 3(c). We show in Fig. 3(d) the $x$ dependence of the sheet carrier density $\left(n_{2 D}\right)$, the longitudinal sheet conductivity $\left(\sigma_{x x}\right)$, and the Hall conductivity $\left(\sigma_{x y}\right)$ at zero fields as converted from $\rho_{x x}$ and $\rho_{y x}$. The notable feature is that the $\sigma_{x y}$ exceeds $0.2 e^{2} / h$ in the most insulating sample $(x=0.6)$, where $\sigma_{x x} \sim 2 e^{2} / h$ and $n_{2 D} \sim$ $10^{12} \mathrm{~cm}^{-2}$ [46]. The sheet carrier densities are estimated from the slope of Hall resistance above the saturation field. The carrier types are electrons for $x=0.3$ and holes for 
$x=0.64$, demonstrating that the Fermi level is systematically shifted from $n$ to $p$ type with increasing $x$ [Fig. 3(c)].

These observations in Fig. 3 can be understood by the opening of an exchange gap in the dispersion relation of the TI surface state. When an exchange gap opens on the surface of the TI, the Berry curvature is strongly enhanced near the band edge, resulting in the large $\sigma_{x y}$. At the same time, when the Fermi energy is tuned within or close to the exchange gap, $\sigma_{x y}$ takes a maximum, while $\sigma_{x x}$ takes a minimum. In the present study, we observe that $\sigma_{x y}$ takes a maximum accompanied by a nearly minimum value of $\sigma_{x x}$ in the sample with the low carrier density $(x=0.6)$ as expected. Also, the decrease in $\sigma_{x y}$ and increase in $\sigma_{x x}$ are observed as the carrier density is detuned from the optimum value. These carrier density dependencies are consistent with the picture described above, indicating the opening of the exchange gap by the magnetic proximity effect. The increase in $\sigma_{x y}$ accompanied by the decrease in $\sigma_{x x}$ leads to an enhancement in the Hall angle $\theta_{H}=\tan ^{-1}\left(\sigma_{x y} / \sigma_{x x}\right)$, discriminating the anomalous Hall effect of extrinsic origin [45]. The obtained values of the $\sigma_{x y}$ and the $\theta_{H}$ are dramatically increased in our samples compared to those reported in other FMI-TI systems [Fig. 3(e)] [34]. This trend suggests that the Fermi level is close to the exchange gap and/or that the exchange gap is large in our samples compared to those FMI-TI systems, although the quantitative estimation of the size of the exchange gap is difficult due to residual disorder and inhomogeneity in the samples $[43,44]$ (see Supplemental Material [34] for the detailed discussion).

The CGT-layer thickness dependence provides additional evidence that the observed anomalous Hall effect is induced by the magnetic proximity effect, excluding other origins arising from $\mathrm{Cr}$ diffusion into the BST layer. Figure 4(a) shows the temperature-dependent magnetization $(M-T)$ curves of four CGT/BST bilayers [inset of Fig. 4(c)] films with representative CGT-layer thicknesses under $\mu_{0} H_{\perp}=0.05 \mathrm{~T}$. Both magnetization $M$ and Curie temperature $T_{C}$ decrease systematically with decreasing the CGT film thickness $t$. As shown in Fig. 4(b), the lowtemperature values of $\sigma_{x y}$ also decrease with decreasing $t$. In Fig. 4(c), the $t$ dependencies of $\sigma_{x y}$ at $T=2 \mathrm{~K}$ and the saturated magnetization of CGT $M_{s}$ are plotted together. The agreement in $t$ dependencies of $\sigma_{x y}$ and $M_{s}$ indicates that $\sigma_{x y}$ is almost proportional to $M_{s}$. This observation shows that the exchange gap on the surface state of the TI can be tuned by the magnetization of the CGT layer, directly pointing to the proximity-coupling origin of the anomalous Hall effect. The decrease in $M_{s}$ in the range of $t<2 \mathrm{~nm}$ is attributed perhaps to the finite size effect of the 2D ferromagnetic CGT layer [31]. In contrast to $\sigma_{x y}, \sigma_{x x}$ is almost constant with variation of $t$ across $t \sim 2 \mathrm{~nm}$ [Fig. 4(c)]. The constancy of $\sigma_{x x}$ suggests that the Fermi energy and the scattering time are not largely affected by the thickness of the CGT layer.
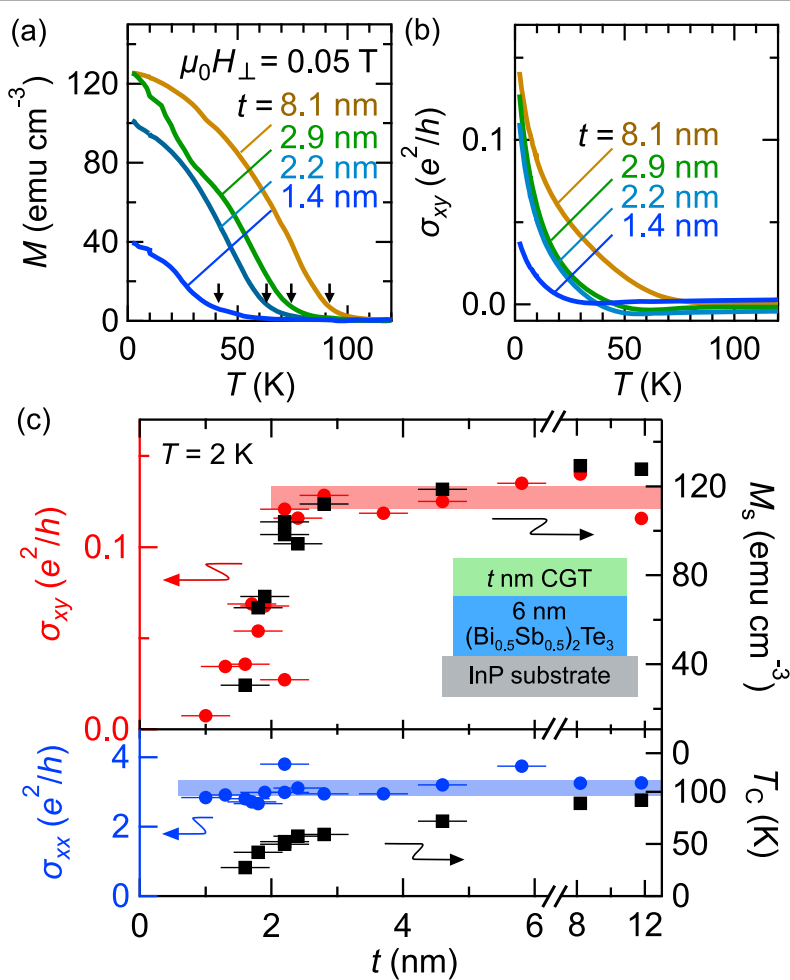

FIG. 4. (a) $M-T$ and (b) $\sigma_{x y}-T$ curves measured under field cooling with $\mu_{0} H_{\perp}=0.05 \mathrm{~T}$ in BST/CGT $(t=1.4,2.2,2.9$, and $8.1 \mathrm{~nm})$ bilayer structures. Black arrows indicate the Curie temperature $T_{C}$ for highlighting the changes against $t$. (c) CGT thickness $t$ dependence of $\sigma_{x y}$ (red closed circles, left red ordinate) and the spontaneous magnetization $M_{s}$ (black closed squares, right black ordinate) at $2 \mathrm{~K}$ under zero magnetic field (top) and $\sigma_{x x}$ at $2 \mathrm{~K}$ (blued closed circles, left blue ordinate) and $T_{C}$ (black closed squares, right black ordinate) (bottom). (Inset) Schematic layout of $\left(\mathrm{Bi}_{0.5} \mathrm{Sb}_{0.5}\right)_{2} \mathrm{Te}_{3}(6 \mathrm{~nm}) / \mathrm{CGT}(t \mathrm{~nm})$ bilayer structure.

On the basis of the above experimental results, we discuss possible mechanisms of the exchange gap formation at the CGT/BST interface. One conceivable scenario would be the induction of magnetization in the TI layer by the adjacent FMI layer as discussed in earlier works $[15-17,25,26,28]$. However, this scenario is unlikely applicable to the present case. At the interfaces of $\mathrm{EuS} / \mathrm{Bi}_{2} \mathrm{Se}_{3}$ and EuS/BST, large magnetizations of about 270 and $160 \mathrm{emu} / \mathrm{cm}^{3}$, respectively, in the TI layer have been reported $[25,26]$. In contrast, in the present study, the magnetization in the CGT layer is already smaller than these values. Therefore, the induced magnetization, even if it existed, in the BST layer of the present CGT/BST heterostructure would be much smaller than that reported for the EuS-based heterostructures [25,26]. Despite the small induced magnetization, our transport measurements have revealed that the $\sigma_{x y}$ and $\theta_{H}$ are much enhanced in the CGT/BST system. One other possible scenario to understand these observations is the formation of the exchange gap by penetration of the TI surface state wave function 
into the FMI layer. In this scenario, even if the interfacial magnetization in the BST layer is small, the penetrated part of the surface state wave function can interact with the magnetic moment in the CGT to produce a sizable exchange gap. A recent first-principles calculation work [29] indicates the formation of a large exchange gap in Tebased heterostructure $\mathrm{MnBi}_{2} \mathrm{Te}_{4} / \mathrm{Bi}_{2} \mathrm{Te}_{3}$ based on the wave function penetration mechanism.

In summary, we have synthesized CGT/BST/CGT heterostructures and have studied the ferromagnetic proximity effect at the interface of the heterostructures. We have observed the depth profile of the magnetization by the PNR measurement, which suggests small induced magnetization in the BST layer. We have also observed a large anomalous Hall angle in magnetotransport measurements, which indicates a sizable exchange gap. To explain both observations, we have proposed the exchange gap formation due to the penetration of the TI surface state wave function into the FMI layer.

We acknowledge helpful discussions with K. Yasuda, R. Watanabe, R. Fujimura, Y. Fujishiro, Y. Okamura, Y. Kaneko, S. Maekawa, J. G. Checkelsky, and N. Nagaosa. This research was partly supported by JSPS/MEXT Grant-in-Aid for Scientific Research (No. 15H05853, No. 15H05867, No. 17J03179, No. 18H04229, No. 18H01155), and JST CREST (No. JPMJCR16F1). This neutron experiment at MLF, J-PARC was performed under a user program (Proposal No. 2017B0195).

*mogi@cmr.t.u-tokyo.ac.jp

†tokura@ riken.jp

[1] M. Z. Hasan and C. L. Kane, Rev. Mod. Phys. 82, 3045 (2010).

[2] X. L. Qi and S.-C. Zhang, Rev. Mod. Phys. 83, 1057 (2011).

[3] X.-L. Qi, T. L. Hughes, and S.-C. Zhang, Phys. Rev. B 78, 195424 (2008).

[4] R. Yu, W. Zhang, H. J. Zhang, S.-C. Zhang, X. Dai, and Z. Fang, Science 329, 61 (2010).

[5] C.-Z. Chang et al., Science 340, 167 (2013).

[6] C.-Z. Chang, W. W. Zhao, D. Y. Kim, H. J. Zhang, B. A. Assaf, D. Heiman, S.-C. Zhang, C. X. Liu, M. H. W. Chan, and J. S. Moodera, Nat. Mater. 14, 473 (2015).

[7] M. Mogi, R. Yoshimi, A. Tsukazaki, K. Yasuda, Y. Kozuka, K. S. Takahashi, M. Kawasaki, and Y. Tokura, Appl. Phys. Lett. 107, 182401 (2015).

[8] Y. Ou, C. Liu, G. Jiang, Y. Feng, D. Zhao, W. Wu, X.-X. Wang, W. Li, C. Song, L.-L. Wang, W. Wang, W. Wu, Y. Wang, K. He, X.-C. Ma, and Q.-K. Xue, Adv. Mater. 30, 1703062 (2018).

[9] M. Mogi, M. Kawamura, R. Yoshimi, A. Tsukazaki, Y. Kozuka, N. Shirakawa, K. S. Takahashi, M. Kawasaki, and Y. Tokura, Nat. Mater. 16, 516 (2017).

[10] M. Mogi, M. Kawamura, A. Tsukazaki, R. Yoshimi, K. S. Takahashi, M. Kawasaki, and Y. Tokura, Sci. Adv. 3, eaao1669 (2017).
[11] D. Xiao, J. Jiang, J.-H. Shin, W. Wang, F. Wang, Y.-F. Zhao, C. Liu, W. Wu, M. H. W. Chan, N. Samarth, and C.-Z. Chang, Phys. Rev. Lett. 120, 056801 (2018).

[12] I. Lee, C. K. Kim, J. Lee, S. J. L. Billinge, R. Zhong, J. A. Schneeloch, T. Liu, T. Valla, J. M. Tranquada, G. Gu, and J. C. Séamus Davis, Proc. Natl. Acad. Sci. U.S.A. 112, 1316 (2015).

[13] E. O. Lachman, A. F. Young, A. Richardella, J. Cuppens, H. R. Naren, Y. Anahory, A. Y. Meltzer, A. Kandala, S. Kempinger, Y. Myasoedov, M. E. Huber, N. Samarth, and E. Zeldov, Sci. Adv. 1, e1500740 (2015).

[14] H. Beidenkopf, P. Roushan, J. Seo, L. Gorman, I. Drozdov, Y. S. Hor, R. J. Cava, and A. Yazdani, Nat. Phys. 7, 939 (2011).

[15] W. Luo and X.-L. Qi, Phys. Rev. B 87, 085431 (2013).

[16] S. V. Eremeev, V. N. Men'shov, V. V. Tugushev, P. M. Echenique, and E. V. Chulkov, Phys. Rev. B 88, 144430 (2013).

[17] P. Wei, F. Katmis, B. A. Assaf, H. Steinberg, P. JarilloHerrero, D. Heiman, and J. S. Moodera, Phys. Rev. Lett. 110, 186807 (2013).

[18] Q. I. Yang, M. Dolev, L. Zhang, J. Zhao, A. D. Fried, E. Schemm, M. Liu, A. Palevski, A. F. Marshall, S. H. Risbud, and A. Kapitulnik, Phys. Rev. B 88, 081407(R) (2013).

[19] A. Kandala, A. Richardella, D. W. Rench, D. M. Zhang, T. C. Flanagan, and N. Samarth, Appl. Phys. Lett. 103, 202409 (2013).

[20] M. Lang, M. Montazeri, M. C. Onbasli, X. Kou, Y. Fan, P. Upadhyaya, K. Yao, F. Liu, Y. Jiang, W. Jiang, K. L. Wong, G. Yu, J. Tang, T. Nie, L. He, R. N. Schwartz, Y. Wang, C. A. Ross, and K. L. Wang, Nano Lett. 14, 3459 (2014).

[21] L. D. Alegria, H. Ji, N. Yao, J. J. Clarke, R. J. Cava, and J. R. Petta, Appl. Phys. Lett. 105, 053512 (2014).

[22] Z. Jiang, C.-Z. Chang, C. Tang, P. Wei, J. S. Moodera, and J. Shi, Nano Lett. 15, 5835 (2015).

[23] C. Tang, C.-Z. Chang, G. Zhao, Y. Liu, Z. Jiang, C.-X. Liu, M. R. McCartney, D. J. Smith, T. Chen, J. S. Moodera, and J. Shi, Sci. Adv. 3, e1700307 (2017).

[24] S. Zhu, D. Meng, G. Liang, G. Shi, P. Zhao, P. Cheng, Y. Li, X. Zhai, Y. Lu, L. Chen, and K. Wu, Nanoscale 10, 10041 (2018).

[25] F. Katmis, V. Lauter, F. S. Nogueira, B. A. Assaf, M. E. Jamer, P. Wei, B. Satpati, J. W. Freeland, I. Eremin, D. Heiman, P. Jarillo-Herrero, and J. S. Moodera, Nature (London) 533, 513 (2016).

[26] M. Li, Q. Song, W. Zhao, J. A. Garlow, T.-H. Liu, L. Wu, Y. Zhu, J. S. Moodera, M. H. W. Chan, G. Chen, and C.-Z. Chang, Phys. Rev. B 96, 201301(R) (2017).

[27] T. Hirahara et al., Nano Lett. 17, 3493 (2017).

[28] V. N. Men'shov, V. V. Tugushev, S. V. Eremeev, P. M. Echenique, and E. V. Chulkov, Phys. Rev. B 88, 224401 (2013).

[29] M. M. Otrokov, T. V. Menshchikova, M. G. Vergniory, I. P. Rusinov, A. Y. Vyazovskaya, Y. M. Koroteev, G. Bihlmayer, A. Ernst, P. M. Echenique, A. Arnau, and E. V. Chulkov, 2D Mater. 4, 025082 (2017).

[30] V. Carteaux, D. Brunet, G. Ouvrard, and G. André, J. Phys. Condens. Matter 7, 69 (1995).

[31] H. Ji, R. A. Stokes, L. D. Alegria, E. C. Blomberg, M. A. Tanatar, A. Reijnders, L. M. Schoop, T. Liang, R. Prozorov, 
K. S. Burch, N. P. Ong, J. R. Petta, and R. J. Cava, J. Appl. Phys. 114, 114907 (2013).

[32] C. Gong, L. Li, Z. Li, H. Ji, A. Stern, Y. Xia, T. Cao, W. Bao, C. Wang, Y. Wang, Z. Q. Qiu, R. J. Cava, S. G. Louie, J. Xia, and X. Zhang, Nature (London) 546, 265 (2017).

[33] M. Mogi, A. Tsukazaki, Y. Kaneko, R. Yoshimi, K. S. Takahashi, M. Kawasaki, and Y. Tokura, APL Mater. 6, 091104 (2018).

[34] See Supplemental Material at http://link.aps.org/ supplemental/10.1103/PhysRevLett.123.016804 for details about thin film growth, polarized neutron reflectometry, and additional transport and magnetization results, which includes Refs. [12,14,17,21-25,33,35-45].

[35] R. Yoshimi, A. Tsukazaki, Y. Kozuka, J. Falson, K. S. Takahashi, J. G. Checkelsky, N. Nagaosa, M. Kawasaki, and Y. Tokura, Nat. Commun. 6, 6627 (2015).

[36] C.-Z. Chang, P. Tang, X. Feng, K. Li, X.-C. Ma, W. Duan, K. He, and Q.-K. Xue, Phys. Rev. Lett. 115, 136801 (2015).

[37] M. Takeda et al., Chin. J. Phys. 50, 161 (2012).
[38] K. Nakajima et al., Quantum Beam Sci. 1, 9 (2017).

[39] Y. Inamura, T. Nakatani, J. Suzuki, and T. Otomo, J. Phys. Soc. Jpn. 82, SA031 (2013).

[40] M. Björck and G. Andersson, J. Appl. Crystallogr. 40, 1174 (2007).

[41] J. Zhang, C.-Z. Chang, Z. Zhang, J. Wen, X. Feng, K. Li, M. Liu, K. He, L. Wang, X. Chen, Q.-K. Xue, X. Ma, and Y. Wang, Nat. Commun. 2, 574 (2011).

[42] R. Dingle, H. L. Störmer, A. C. Gossard, and W. Wiegmann, Appl. Phys. Lett. 33, 665 (1978).

[43] B. Skinner, T. Chen, and B. I. Shklovskii, Phys. Rev. Lett. 109, 176801 (2012).

[44] N. A. Sinitsyn, J. E. Hill, H. Min, J. Sinova, and A. H. MacDonald, Phys. Rev. Lett. 97, 106804 (2006).

[45] N. Nagaosa, J. Sinova, S. Onoda, A. H. MacDonald, and N. P. Ong, Rev. Mod. Phys. 82, 1539 (2010).

[46] The $n_{2 D}$ at $x=0.6$ is slightly higher than that at $x=0.5$. We speculate that this can be related to the coexistence of electrons and holes near the charge neutrality condition. 